\title{
Increased interleukin-6 levels in neuron-derived plasma small extracellular vesicles of subarachnoid haemorrhage patients
}

\section{Niansheng Lai}

The First Affiliated Hospital of Wannan Medical College

\section{Yang Yao}

The First Affiliated Hospital of Wannan Medical College

Tao Yu

The First Affiliated Hospital of Wannan Medical College

\section{Dayong Xia}

The First Affiliated Hospital of Wannan Medical College

\section{Xintong Zhao}

The First Affiliated Hospital of Wannan Medical College

\section{Degang Wu}

The First Affiliated Hospital of Wannan Medical College

\section{Zhenbao Li}

The First Affiliated Hospital of Wannan Medical College

\section{Xinggen Fang}

The First Affiliated Hospital of Wannan Medical College

Bin Sheng ( $\nabla$ mshengbin@126.com )

the first affiliated hosptial of wannan medical college https://orcid.org/0000-0001-6974-0195

\section{Research}

Keywords: Subarachnoid hemorrhage, Small extracellular vesicles, Interleukin-6, Biomarker

Posted Date: October 7th, 2020

DOI: https://doi.org/10.21203/rs.3.rs-83150/v1

License: (c) (1) This work is licensed under a Creative Commons Attribution 4.0 International License. Read Full License 


\section{Abstract}

Background: Aneurismal subarachnoid hemorrhage (aSAH) is a serious type of stroke with high mortality and disability. Identifying circulating biomarkers is helpful to improve theranostics of aSAH. In this study, we are for the first time to report circulating interleukin-6(IL-6) in neuron-derived small extracellular vesicles(NDSEVs) were identified to be the potential biomarkers in prognosis of aSAH.

Methods: We extracted small extracellular vesicles from the plasma of aSAH patients and healthy controls and were enriched by sequential precipitation and anti-L1CAM antibody immunoabsorption. Subsequently,we determined IL-6 levels by an enzyme-linked immunosorbent assay (ELISA).

Result: Plasma IL-6 NDSEVs showed distinct pattern differences between aSAH patients and healthy controls. The IL- 6 NDSEVs levels were increased and positively associated with disease monitoring and prognosis of aSAH patients. These data suggest an elevated neuroinflammatory cascade in aSAH patients.

Conclusion: The IL-6 NDSEVs maybe prospective biomarkers to indicate its progression, and thus may own great potential in applications such as prognostic evaluation of aSAH in the near future.

\section{Background}

Aneurysmal subarachnoid hemorrhage (aSAH) typically results from a ruptured aneurysm and is a clinical syndrome with $45 \%$ mortality and disability with morbidity of approximately 6-16 per 100000 individuals every year all around the world, which occured at youny age and accounted for $5-7 \%$ of the total incidence of stroke[1, 2]. A major prognostic determinant is the characteristics of the initial hemorrhage, which causes early brain injury (EBI) and early cerebral vasospasms, and may be associated with delayed cerebral ischaemia (DCI)[3]. Recently, researchers have increasingly found that EBI after SAH may be the leading factor contributing to an unfavourable outcome of SAH patients $[4,5]$. Therefore, the importance of pathophysiologic mechanisms in the very early phase after aSAH with changes including microvascular filling defects, breakdown of ionic homeostasis, inflammation, and microarterial narrowing[6-8]. Moreover, a reliable, early, economic and non-invasive approach is urgently in need to provide screening to patients in order to improve the prognosis of patients with aSAH.

Accumulating studies revealed small extracellular vesicles (sEVs), which are lipid membrane vesicles, could cross blood-brain barrier(BBB) and actually specialized in long distance intercellular communications facilitating transfer of proteins, lipids, and nucleic acid for subsequent protein expression in target cells $[9,10]$. sEVs also released by brain cells are able to cross the BBB and can be detected in blood circulating[11-13]. Similarly, endothelial and peripheral cells secrete sEVs into the circulation. sEVs can be enriched from peripheral blood samples and used for detection of various proteins, lipids, and nucleic acids[14]. Together, these circulating sEVs could potentially be ideal biomarkers to reflect the pathological progress of aSAH. Several clinical studies have examined 
circulating sEVs contents, including functional proteins and various nucleic acid species as biomarkers for cerebral ischemia[15-19].

There is substantial evidence that the inflammatory response occured early after SAH and contributed to the progression of SAH-induced EBI[4, 8, 20]. Potential biomarkers that have been studied of inflammatory cytokines such as interleukin-1a(IL-1a), IL-1 $\beta$, IL-6, IL-8,IL-18, and tumor necrosis factoralpha[21-23]. IL-6 is a proinflammatory cytokine in response to acute brain injury and other diseases[17, $24,25]$. Several studies have demonstrated elevated IL-6 levels may induce neuroinflammation and may be closely associated with the prognosis of aSAH patients[23, 26, 27].

Therefore, we hypothesize that aSAH leads to changes in the expression of IL-6 in sEVs in the brain and that these NDSEVs are secreted into circulating where they may serve as biomarkers for aSAH.In the present study, we extracted NDSEVs from the plasma of aSAH patients and healthy controls to determine the expression of IL- 6 levels in NDSEVs and, ultimately, we detected increased IL- 6 levels in NDSEVs of aSAH patients, which evaluated possible associations between the markers of early inflammatory response and disease progression.

\section{Methods}

\section{Ethics}

Study participants were recruited from the Department of Neurosurgery, The First Affiliated Hospital of Wannan Medical College, Wuhu City, China. The study was performed in accordance with the Declaration of Helsinki. Written informed consent was received from participants or valid proxies (family or a professional not directly involved in the study) prior to inclusion in the study. All experiments were approved by the Ethics Committee of the First Affiliated Hospital of Wannan Medical College.

\section{Study design}

The aSAH patients included in the present study were admitted from May 2016 to March 2017. One hundred -seventeen patients with aSAH within the $24 \mathrm{~h}$ after SAH, out of a total of 224 patients with SAH during this period, were collected plasma. The mean age \pm SD was $59.91 \pm 9.65$ years (range, $40-$ 83 year); there were 72 women and 45 men.Exclusion criteria were as follows: Admission later than $72 \mathrm{~h}$ after onset of bleeding; non-aneurysmal SAH; with liver, kidney, heart or lung insufficiency or infectious diseases; poor prognosis upon admission without any intervention. Age-matched health persons in the fasting state were used as controls $(n=40)$.

Initial clinical status was accessed was using the World Federation of Neurological Surgeons (WFNS) grade [28]. The amount of blood in computed tomography (CT) was assessed using the modified Fisher' scale. All patients were treated with endovascular coiling. Neurologic outcome was assessed using the Modified Rankin Scale (mRS) at 1-year post-aSAH by structured telephone interview[29]. If no contact was obtained after this procedure, the patient was declared lost to follow-up. At the end of the follow-up 
period, patients with a mRS score of $0-2$ were classified as having a good outcome, and those with a mRS score of 3-6 were classified as having a poor outcome. Table 1 and Table 2 summarizes the basic characteristics of the population.

Table 1

Characteristics and clinical data of study population

\begin{tabular}{|c|c|c|c|}
\hline Parameters & aSAH group,n = 117 & Control group, $\mathrm{n}=40$ & $P$ value \\
\hline Sex; Male & 45 & 16 & \multirow[t]{2}{*}{0.85} \\
\hline Sex; Female & 72 & 24 & \\
\hline Age;mean \pm SD (years) & $59.91 \pm 9.65$ & $57.03 \pm 9.07$ & 0.10 \\
\hline Hypertension; Yes & 49 & 15 & \multirow[t]{2}{*}{0.71} \\
\hline Hypertension; No & 68 & 25 & \\
\hline Smoking; Yes & 40 & 11 & \multirow[t]{2}{*}{0.56} \\
\hline Smoking; No & 77 & 29 & \\
\hline $\mathrm{IL}-6_{\mathrm{NDSEV}} \pm \mathrm{SD}(\mathrm{pg} / \mathrm{mL})$ & $365.71 \pm 44.57$ & $8.81 \pm 1.63$ & $<0.001$ \\
\hline
\end{tabular}


Table 2

Clinicopathological features of aSAH

\begin{tabular}{|c|c|c|c|}
\hline Parameters & $\mathrm{mRS} 0-2, \mathrm{n}=78$ & mRS 3-6,n = 39 & $P$ value \\
\hline Sex; Male & 30 & 15 & \multirow[t]{2}{*}{1.00} \\
\hline Sex; Female & 48 & 24 & \\
\hline Age; mean \pm SD (years) & $58.78 \pm 9.11$ & $62.15 \pm 10.40$ & 0.075 \\
\hline Hypertension; Yes & 32 & 17 & \multirow[t]{2}{*}{0.84} \\
\hline Hypertension; No & 46 & 22 & \\
\hline Smoking; Yes & 25 & 15 & \multirow[t]{2}{*}{0.54} \\
\hline Smoking; No & 53 & 24 & \\
\hline Diabetes;Yes & 12 & 7 & \multirow[t]{2}{*}{0.80} \\
\hline Diabetes;No & 65 & 33 & \\
\hline Alcohol abuse;Yes & 7 & 7 & \multirow[t]{2}{*}{0.23} \\
\hline Alcohol abuse;No & 71 & 32 & \\
\hline \multicolumn{4}{|l|}{ Aneurysm position } \\
\hline $\mathrm{ACA}+\mathrm{AComA}$ & 21 & 15 & \multirow[t]{4}{*}{0.52} \\
\hline $\mathrm{ICA}+\mathrm{PComA}$ & 27 & 12 & \\
\hline MCA & 19 & 6 & \\
\hline Vertebrobasilar system & 11 & 6 & \\
\hline Aneurysm diameter; mean $\pm \mathrm{SD}(\mathrm{mm})$ & $5.72 \pm 2.15$ & $8.16 \pm 3.62$ & $<0.001$ \\
\hline WFNS Grade ; I & 14 & 1 & \multirow[t]{5}{*}{$<0.001$} \\
\hline WFNS Grade ; II & 30 & 6 & \\
\hline WFNS Grade ; III & 19 & 2 & \\
\hline WFNS Grade ; IV & 15 & 22 & \\
\hline WFNS Grade ; V & 0 & 8 & \\
\hline \multicolumn{4}{|l|}{ Modified Fisher Score } \\
\hline \multicolumn{3}{|l|}{ I: No thick cisternal blood, -IVH } & $<0.001$ \\
\hline \multicolumn{4}{|c|}{$\begin{array}{l}\text { ACA Anterior cerebral artery; AComA Anterior communicant artery; DCI Delayed cerebral ischemia; ICA } \\
\text { Internal carotid artery; MCA Middle cerebral artery; mFS Modified Fisher Score; mRS Modified Rankin } \\
\text { Scale; NDSEVs Neuron-derived plasma small extracellular vesicles; PComA Posterior communicant } \\
\text { artery; SD Standard deviation; WFNS World Federation of Neurological Surgeons ; }\end{array}$} \\
\hline
\end{tabular}




\begin{tabular}{|c|c|c|c|}
\hline Parameters & $\mathrm{mRS} 0-2, \mathrm{n}=78$ & mRS 3-6,n = 39 & $P$ value \\
\hline Il:No thick cisternal blood,+IVH & 14 & 0 & \\
\hline III:Thick cisternal blood, -IVH & 39 & 14 & \\
\hline IV:Thick cisternal blood,+IVH & 20 & 25 & \\
\hline Acute hydrocephalus; Yes & 11 & 18 & $<0.001$ \\
\hline Acute hydrocephalus; No & 67 & 21 & \\
\hline DCl; Yes & 12 & 23 & $<0.001$ \\
\hline DCl; No & 66 & 16 & \\
\hline $\mathrm{IL}-6_{\mathrm{NDSEV}} \pm \mathrm{SD}(\mathrm{pg} / \mathrm{mL})$ & $342.24 \pm 29.48$ & $412.65 \pm 30.18$ & $<0.001$ \\
\hline \multicolumn{4}{|c|}{$\begin{array}{l}\text { ACA Anterior cerebral artery; AComA Anterior communicant artery; DCI Delayed cerebral ischemia; ICA } \\
\text { Internal carotid artery; MCA Middle cerebral artery; mFS Modified Fisher Score; mRS Modified Rankin } \\
\text { Scale; NDSEVs Neuron-derived plasma small extracellular vesicles; PComA Posterior communicant } \\
\text { artery; SD Standard deviation; WFNS World Federation of Neurological Surgeons; }\end{array}$} \\
\hline
\end{tabular}

\section{Sample processing}

The blood samples were processed for plasma isolation within $2 \mathrm{~h}$ after withdrawal using BD EDTAtreated plasma separator tubes. Whole blood was centrifuged at $500 \mathrm{rpm}$ and $4{ }^{\circ} \mathrm{C}$ for $10 \mathrm{~min}$. And the upper-layer was transferred into a RNase/DNase - free $1.5 \mathrm{~mL}$ EP tubes followed by further centrifugation at $3000 \mathrm{rpm}$ and $4{ }^{\circ} \mathrm{C}$ for $10 \mathrm{~min}$. Plasma was divided into aliquots and stored at $-80^{\circ} \mathrm{C}$ for further analysis.

\section{Isolation of NDSEVs from plasma samples}

sEVs were isolated using miRCURY Exosome Kits (from Cell/Urine/CSF; Qiagen, Valencia, CA) following the manufacturer's protocol. Briefly, 400 $\mu \mathrm{L}$ of the Precipitation Buffer B was added to $1 \mathrm{~mL}$ of plasma samples and mixed well for 60 minutes at $4{ }^{\circ} \mathrm{C}$, followed by centrifugation at $10,000 \mathrm{~g}$ for 30 minutes at room temperature. The sEVs were harvested by removing the supernatant. The sEVs were resuspended in $100 \mu \mathrm{L}$ of Resuspension Buffer. To enrich NDSEVs, total sEVs were harvested by immunoabsorption enrichment with mouse anti-human CD171(L1CAM neural adhesion protein) biotinylated antibody ( eBiosciences, San Diego, CA, USA) as described[11]. Then NDSEVs were lysed by $1 \%$ Triton X-100 lysis buffer(Beyotime, P0013) that contained protease and phosphatase inhibitor cocktails. The lysates were stored at $-80^{\circ} \mathrm{C}$.

\section{Exosomes identification}

For NDSEVs identification, transmission electron microscopy (TEM, Hitachi HT7700, Tokyo, Japan) was performed to observe the morphology of precipitated particles. Briefly, we extracted previous eluates of $5 \mu \mathrm{l}$ and diluted them to $10 \mu \mathrm{l}$. Then, we extracted $10 \mu \mathrm{l}$ sample onto copper disks for $1 \mathrm{~min}$ and use filter paper to remove the floating material. Subsequently, $10 \mu$ phosphotungstic acid was dropped on copper 
disks for 1 min and the floating material was removed using filter paper. After drying for severe minutes and finally examined with a JEOL JEM-1400 transmission electron microscope at $80 \mathrm{kV}$. The size distribution and concentration of the particles was measured and analysed using NanoFCM instrument (NanoFCM Inc. China). Biomarkers for exosomes CD9, CD81 were also measured using the NanoFCM instrument.

\section{Isolation and measure concentration of exosomes protein}

The bicinchoninic acid ( $\mathrm{BCA}$ ) method was used to measure the concentration of exosomes using the enhanced BCA protein assay kit (Beyotime, P0010). Briefly, the isolated NDSEVs were melted at $37^{\circ} \mathrm{C}$. We quickly added equal volumes of RIPA lysis buffer, then mixed and split on ice for $30 \mathrm{~min}$. A standard sample of BCA method protein concentration was formulated and then added to the BCA mixture and mixed. After incubating $30 \mathrm{~min}$ at $37^{\circ} \mathrm{C}$, optical density was measured at OD560 $\mathrm{nm}$ on a microplate reader. The protein concentration of the sample was calculated according to the standard curve.

\section{Western blot analysis}

Molecular Weight maker (5 IL/lane; Thermos Scientific, Waltham, MA, USA) and protein samples (20 $\mathrm{lg} /$ lane) were separated using a $10 \%$ sodium dodecyl sulfate-polyacrylamide gel(SDS-PAGE), and electrophoretically transferred onto a polyvinylidene difluoride membrane (PVDF, Immobilon-PSQ, Millipore Corporation, Billerica, MA, USA). Then, the membrane was blocked with $5 \%$ non-fat milk for $1 \mathrm{~h}$ at room temperature. Blots were incubated with primary antibodies in 5\% BSA (in PBS + 0.1\% Tween 20) overnight at $4{ }^{\circ} \mathrm{C}$. Mouse anti-CD9, CD81 and rabbit anti-alix (all 1:1,000, Abcam) were used as primary antibodies. Then corresponding HRP-conjugated anti-rabbit, or anti-mouse $(1: 10,000$, Pierce) secondary antibodies were incubated for another $2 \mathrm{~h}$ under the same conditions. Bands were visualized with an enhanced chemiluminescence (ECL) kit(Beyotime).

\section{Flow nano analyser studies}

We diluted $20 \mu \mathrm{L}$ NDSEVs to $60 \mu \mathrm{L}$, then added $20 \mu \mathrm{L}$ fluorescent markers antibodies (CD9, CD81) to $30 \mu \mathrm{L}$ dilution. We incubated for $30 \mathrm{~min}$ at $37^{\circ} \mathrm{C}$ after mixing. We added $1 \mathrm{ml}$ pre-cooled PBS, then ultracentrifuged for 70 min with $110,000 \mathrm{xg}$ at $4^{\circ} \mathrm{C}$. After removing supernatants, we repeated centrifugation. We removed supernatant and resuspended in $50 \mu \mathrm{L}$ pre-cooled 1 x PBS and analysed using the NanoFCM as per manufacturer's instructions.

\section{Enzyme-linked immunosorbent assay (ELISA)}

The concentrations of IL-6, and tetra-spanning exosome marker CD81 in NDSEVs were quantified using specific ELISA kits (Elabscience Biotechnology, China) according to the manufacturer's instructions. The mean value for all determinations of CD81 in the patient cohort was set at 1.00 and relative values for each specimen were used to normalize their recovery. The final concentration of cytokines was measured using OD values. One laboratory technicians measured all ELISAs without knowledge of the clinical information. 


\section{Statistical analysis}

All data were analyzed using MedCalc version 15.0.0 (Medcalc Software bvba, Ostend, Belgium). Data were presented as mean \pm standard deviation (SD). The Mann-Whitney $U$ test was used to assess the differences between two groups and the Kruskal-Wallis test for differences between more than two groups. The correlations among the variables were calculated using Spearman rank correlation coefficient analysis. For comparison of levels of IL- 6 over time between patients with poor and good outcome, a generalized linear relationship model with Spearman's correlation coefficient was used. Receiver operating characteristic(ROC) curves were constructed to determine the optimal thresholds of IL6 for aSAH. A logistic regression model was analyzed to determine factors that predicted the mRS after adjusting for risk factors that reached $P<0.1$ in the univariate analyses.A P-value of $<0.05$ was considered significant.

\section{Results}

\section{Patient characteristics}

In this pilot study, a total of 117 aSAH patients and 40 health controls were recruited. Study design was followed as Fig. 1.Plasma was taken at $24 \mathrm{~h}$ after $\mathrm{SAH}$, and obtained in the fasting state in each healthy control. The detailed characteristics for these two groups are summarized in Table 2.

\section{NDSEVs characterization}

The morphology of NDSEVs was evaluated using TEM. Exosomes had spherical shapes with sizes of $86.71 \pm 16.82 \mathrm{~nm}$ surrounded by membranes (Fig. 2A and 2B). The STD concentration of exosomes was $6.09 \mathrm{E}+8$ particles $/ \mathrm{mL}$ (Fig. 2C). Exosome protein concentration was determined using the BCA method.

The OD $560 \mathrm{~nm}$ was 0.231 and the protein concentration was $1.23 \mu \mathrm{g} / \mu \mathrm{L}$ according to the standard curve (Fig. 2D). The identity of exosomes was further validated by quantitating the exosome membraneassociated markers CD9 and CD81 using the NanoFCM. The positivity rates were $5.4 \%$ and $5.7 \%$ (Fig. 2E and $2 \mathrm{~F}$ ). There were no apparent differences in size or shape of exosomes between the control and aSAH samples.

\section{IL-6 in NDSEVs of aSAH patients and healthy controls}

The expression levels of IL- 6 were determined in 117 aSAH patients and 40 healthy controls. The CD81normalized levels of IL- 6 in NDSEVs were significantly increased at 1 day post-aSAH when compared with healthy controls(aSAH: $365.71 \pm 44.57$ pg/ml; Controls: $8.81 \pm 1.63$ pg/ml; $\mathrm{P}<0.001$ )( Fig. 3).

\section{Relationships of IL-6 NDSEVs with aSAH severity}


The WFNS grade is a tool that is used to assess the level of early brain injury after SAH. The modified Fisher' scale was used to assess the amount of blood in CT images of aSAH patients. At the beginning of the acute period, the patients with a WFNS grade of I-III were classified as mildly (mild aSAH), and those with a WFNS grade of $\mathbb{B}-\mathrm{V}$ were classified as severely (severe aSAH). As illustrated in Fig. 4A, comparison of the severe aSAH patients with the mild revealed that the IL- 6 levels significantly increased(Severe aSAH: $409.14 \pm 27.06 \mathrm{pg} / \mathrm{ml}$; mild aSAH: $338.56 \pm 29.15 \mathrm{pg} / \mathrm{ml})(\mathrm{P}<0.001)$. To evaluate the utility of IL-6 NDSEVs for discrimination of severe aSAH from mild aSAH, we performed ROC curve analysis and found that the area under curves(AUCs) of IL-6 NDSEVs was 0.961 (95\% Cl: $0.909-0.988 ; \mathrm{P}<0.001)$ (Fig. 4B). The highest accuracy was at a cutoff expression value of 375.18 , where the positive predictive value, negative predictive value, sensitivity, and pecificity to identify a severe aSAH were $91.1,94.4,91.11$, and $94.44 \%$, respectively.

To further investigate the relationships between the expression levels of IL-6 ${ }_{\text {NDSEVs }}$ and WFNS grade, Spearman's correlation coefficient analysis was performed. The results revealed that in the aSAH patients, the level of IL- 6 NDSEVS $_{\text {s }}(\rho=0.845 ; 95 \%$ Cl: 0.753 to $0.890 ; P<0.001)$ was closely correlated with aSAH severity as scored by WFNS grade.

\section{Relationships of IL-6 NDSEVs with clinical outcomes of aSAH patients}

It is important to determine the potential outcomes of aSAH patients at the earliest stage to optimize the treatment. Thus, the patients were divided into two groups according to their clinical outcomes. At the end of the follow-up period, patients with a mRS score of 0-2 were classified as having a good outcome, and those with a mRS score of 3-6 were classified as having a poor outcome. The mRS scores were lower in the patients with lower expression of IL- $6_{\text {NDSEVs }}$ than in those with higher expression $(P<0.001$; Fig. 5$)$. On univariate analysis, age,WFNS grade, modified Fisher score and IL- 6 NDSEVs levels were identified as prognostic factors 1 year post-SAH $(P<0.1)$.In the logistic regression models, the levels of IL-6 NDSEVs were significantly correlated with mRS score at 1 year post-SAH $(P<0.001 ;$ Table 3$)$. Therefore, outcome prediction may be improved by determination of the expression of IL-6 in. 
Table 3

Multivariable logistic analyses of risk factors in patients with aSAH at 1-year

\begin{tabular}{|llll|}
\hline Parameter & OR & $95 \% \mathrm{Cl}$ & $P$ value \\
\hline Age, per year & 1.17 & 1.02 to 1.35 & 0.024 \\
\hline Aneurysm diameter $>6.5 \mathrm{~mm}$ & 16.52 & 0.999 to 273.08 & 0.050 \\
\hline WFNS Grade & 0.092 & 0.0075 to 1.13 & 0.062 \\
\hline Modified Fisher Score & 0.14 & 0.012 to 1.58 & 0.11 \\
\hline Acute hydrocephalus & 0.0043 & 0.0001 to 0.29 & 0.011 \\
\hline DCl & 4.63 & 0.50 to 42.89 & 0.18 \\
\hline IL-6 $6_{\text {NDSEVs }}$ levels & 1.30 & 1.11 to 1.52 & 0.0013 \\
\hline $\begin{array}{l}\text { The median of aneurysm diameter was 6.5 mm; Cl confidence interval; OR odds ratio; WFNS World } \\
\text { Federation of Neurological Surgeons;MFS Modified Fisher Score }\end{array}$ & \\
\hline
\end{tabular}

\section{Discussion}

In the present study, firstly, the circulating contains in aSAH patients and healthy controls. Secondly, we found that the circulating IL- 6 NDSEVs showed distinct pattern differences between aSAH patients and healthy controls. Thirdly, the circulating IL-6 NDSEVs were higher in patients with higher WFNS grade than in those with lower WFNS grade. Finally, the circulating IL-6 NDSEVs $_{\text {s }}$ were associated with prognosis in aSAH patients.

Several inflammatory cytokines have been found to be linked to aSAH[21]. As a well-known cytokine, IL-6 play an important role in brain injury, and associated with aSAH in numerous studies[23, 26, 30]. Previous study showed that IL-6 in cerebrospinal fluid (CSF) seem to be a biomarker for predicting vasospasm after SAH [31]. Previous studies also showed that IL-6 levels in CSF was associated with prognosis in aSAH patients[21, 27, 30]. Additionally, elevated IL-6 levels in CSF may induce neuroinflammation and may be closely associated with the progression of delayed ischemic neurological deficits after SAH[25]. Furthermore, IL- 6 and TNF-a in CSF could be important biomarkers for early diagnosis and disease monitoring in SAH patients[22]. However, another study reported that CSF IL-6 could be a biomarker of the ventricular infection[32]. In addition, previous study showed that IL-6 in CSF could be a useful diagnostic tool for predicting shunt dependency in aSAH patients with acute hydrocephalus[33]. Serum IL-6 was elevated, and was associated with prognosis in aSAH patients[26, 34]. However, many previous studies showed that determining the IL-6 levels in blood circulation may not be a good way[30, 35]. The circulating could directly reflect the situation of the brain, and peripheral factors might have little effect on cytokines in NDSEVs. Therefore, the measurement of IL-6 levels in CNS-derived sEVs, compared with blood or CSF, may be better to illuminate the actual role of IL-6 in aSAH. Hence, in our study, We analysed whether IL-6 ${ }_{\text {NDSEVs }}$ could serve as biomarkers between aSAH patients and healthy controls. 
The important findings of our study were that IL- 6 NDSEVs levels elevated in aSAH patients and could be a biomarker for the prognosis of aSAH. IL-6 NDSEVs expression may be used to discriminate severe aSAH from mild aSAH. ROC curves with AUC values were constructed. The sensitivity and specificity shown in ROC curve were actually high for IL- 6 NDSEVs levels. Furthermore, the WFNS grade and IL-6 NDSEVs were good predictors of neurologic outcome, whereas age or modified Fisher score were not. These results suggest that IL-6 may participate in the occurrence, development and repair progression of aSAH. However, the others CNS diseases is also associated with the neuroinflammation. Actually, it has been reported that inflammatory cytokines in NDSEVs elevated in traumatic brain injury[11]. Additionally, increased IL-6 Levels in the astrocyte-derived exosomes could be useful to reveal neuroinflammation in sporadic amyotrophic lateral sclerosis patients[36]. Thus, the IL-6 NDSEVs may not be suitable to help distinguish aSAH from other CNS diseases.

Although the present study aimed at investigating the IL-6 ${ }_{\text {NDSEV }}$ levels as potential biomarkers correlated with disease severity and prognosis in aSAH patients, there were a few methodological limitations. First, these was a one single-center study with a wide age range, a small number of patients, and a diverse grade of severity of aSAH with WFNS grades I-V. Second, the samples were collected from patients after surgery and during drug therapy. The surgical treatment and drugs may induce changes in the expression levels of IL-6 NDSEVS.

\section{Conclusion}

In summary, IL-6 NDSEV $_{\text {s }}$ may be a reliable biomarker associated with EBI of aSAH. IL-6 ${ }_{\text {NDSEVs }}$ could be another interesting predictor with clinical outcomes. The indicator may be an early outcome predictor for patients with aSAH,but not the independent one. Future studies are critical to determine if IL-6 NDSEVs plays a regulatory role in disease progression of aSAH and evaluate its potential as a therapeutic target.

\section{Abbreviations}

aSAH: Aneurysmal subarachnoid hemorrhage; AUCs: Area under curves; BBB: Blood-brain barrier; BCA: Bicinchoninic acid; CNS: Central nervous system; CSF: cerebrospinal fluid; CT: Computed tomography; CVs: Cerebral vasospasm; DCl: Delayed cerebral ischemia; EDTA: Ethylene Diamine Tetraacetic Acid; ELISA: Enzyme-linked immunosorbent assay; EBI: Early brain injury; ECL: Enhanced chemiluminescence; IL-6: Interleukin-6; mRS: Modified Rankin Scale; NDSEVs: Neuron-derived small extracellular vesicles; ROC: Receiver operating characteristic; SD: Standard deviation; SDS-PAGE: Sodium dodecyl sulfatepolyacrylamide gels; TEM: Transmission electron microscopy; WFNS: World Federation Of Neurosurgical Societies.

\section{Declarations}

Acknowledgements 
The authors thank all participants and cooperating clinicians, and the department of the Central Laboratory of First Affiliated Hospital of Wannan Medical College for the support of basic facilities and Jiaqi Zhang and Bingbing Zhang for assistance with the sample collection.

\section{Authors' contributions}

Niansheng Lai,Yang Yao conducted experiments and drafted the manuscript. Tao Yu, Dayong Xia, Xintong Zhao and Degang Wu contributed to the experiment design and statistical analysis. Bin Sheng, Xinggen Fang and Zhenbao Li designed the experiments and revised the manuscript.

\section{Funding}

This work was supported by grants from the National Natural Science Foundation of China (no.81701357), Key Research and Development Program of Anhui province (no.201904a07020034), Funding of "Peak" Training Program for Scientific Research of First Affiliated Hospital of Wannan Medical College (no.GF2019G05), Anhui Province's (no.1708085QH181), Natural Science Research Project in Higher Education of Anhui Province (no.KJ2018A0253), Science Research Project of Professional Personnel of First Affiliated Hospital of Wannan Medical College(no.YR201911) and the Young and Middle-Aged Natural Science Foundation of Wannan Medical College (no.WK2019F37).

\section{Availability of data and materials}

The datasets used and/or analyzed during the current study are available from the corresponding author on reasonable request.

\section{Ethics approval and consent to participate}

The procedures in this study approved by the Ethics Committee of the First Affiliated Hospital of Wannan Medical College. All experiments were written informed consent was received from participants or valid proxies (family or a professional not directly involved in the study) prior to inclusion in the study.

\section{Consent for publication}

Not applicable.

\section{Competing interests}

The authors declared no potential conflicts of interest with respect to the research, authorship, and/or publication of this article.

\section{References}

1. Vivancos J, Gilo F, Frutos R, Maestre J, Garcia-Pastor A, Quintana F, et al. Clinical management guidelines for subarachnoid haemorrhage. Diagnosis and treatment. Neurologia. 2014;296:353-70. 
2. Lai NS, Zhang JQ, Qin FY, Sheng B, Fang XG, Li ZB. Serum microRNAs are non-invasive biomarkers for the presence and progression of subarachnoid haemorrhage. Biosci Rep. 2017;371:BSR20160480.

3. Sheng B, Fang X, Liu C, Wu D, Xia D, Xu S, et al. Persistent High Levels of miR-502-5p Are Associated with Poor Neurologic Outcome in Patients with Aneurysmal Subarachnoid Hemorrhage. World Neurosurg. 2018;11610:e92-9.

4. Dou Y, Shen H, Feng D, Li H, Tian X, Zhang J, et al. Tumor necrosis factor receptor-associated factor 6 participates in early brain injury after subarachnoid hemorrhage in rats through inhibiting autophagy and promoting oxidative stress. J Neurochem. 2017;1423:478-92.

5. Witsch J, Kuohn L, Hebert R, Cord B, Sansing L, Gilmore EJ, et al. Early Prognostication of 1-Year Outcome After Subarachnoid Hemorrhage: The FRESH Score Validation. J Stroke Cerebrovasc Dis. 2019;2810:104280.

6. Kessler RA, Garzon-Muvdi T, Yang W, Weingart J, Olivi A, Huang J, et al. Metastatic Atypical and Anaplastic Meningioma: A Case Series and Review of the Literature. World Neurosurg. 2017;101:4756.

7. Rass V, Helbok R, Early Brain Injury After Poor-Grade Subarachnoid Hemorrhage. Curr Neurol Neurosci Rep, 2019; 1910: 78.

8. Gris T, Laplante P, Thebault P, Cayrol R, Najjar A, Joannette-Pilon B, et al. Innate immunity activation in the early brain injury period following subarachnoid hemorrhage. J Neuroinflammation. 2019;161:253.

9. Ramirez SH, Andrews AM, Paul D, Pachter JS. Extracellular vesicles: mediators and biomarkers of pathology along CNS barriers. Fluids Barriers CNS. 2018;151:19.

10. Thery C, Witwer KW, Aikawa E, Alcaraz MJ, Anderson JD, Andriantsitohaina R, et al. Minimal information for studies of extracellular vesicles 2018 (MISEV2018): a position statement of the International Society for Extracellular Vesicles and update of the MISEV2014 guidelines. J Extracell Vesicles. 2018;71:1535750.

11. Goetzl EJ, Peltz CB, Mustapic M, Kapogiannis D, Yaffe K. Neuron-Derived Plasma Exosome Proteins after Remote Traumatic Brain Injury J Neurotrauma. 2020;372:382-8.

12. Ohmichi T, Mitsuhashi M, Tatebe H, Kasai T, Ali El-Agnaf OM, Tokuda T. Quantification of brainderived extracellular vesicles in plasma as a biomarker to diagnose Parkinson's and related diseases. Parkinsonism Relat Disord. 2019;61:82-7.

13. Otero-Ortega L, Laso-Garcia F, Gomez-de Frutos M, Fuentes B, Diekhorst L, Diez-Tejedor E, et al. Role of Exosomes as a Treatment and Potential Biomarker for Stroke. Transl Stroke Res. 2019;103:241-9.

14. Kanninen KM, Bister N, Koistinaho J, Malm T. Exosomes as new diagnostic tools in CNS diseases. Biochim Biophys Acta. 2016;18623:403-10.

15. Li DB, Liu JL, Wang W, Luo XM, Zhou X, Li JP, et al. Plasma Exosomal miRNA-122-5p and miR-300-3p as Potential Markers for Transient Ischaemic Attack in Rats. Front Aging Neurosci. 2018;10:24. 
16. Wang W, Li DB, Li RY, Zhou X, Yu DJ, Lan XY, et al., Diagnosis of Hyperacute and Acute Ischaemic Stroke: The Potential Utility of Exosomal MicroRNA-21-5p and MicroRNA-30a-5p. Cerebrovasc Dis, 2018; 455-6: 204-212.

17. Kerr N, Garcia-Contreras M, Abbassi S, Mejias NH, Desousa BR, Ricordi C, et al. Inflammasome Proteins in Serum and Serum-Derived Extracellular Vesicles as Biomarkers of Stroke. Front Mol Neurosci. 2018;11:309.

18. Yu P, Chen W. Advances in the diagnosis of exosomal miRNAs in ischemic stroke. Neuropsychiatr Dis Treat. 2019;15:2339-43.

19. Zhou J, Chen L, Chen B, Huang S, Zeng C, Wu H, et al. Increased serum exosomal miR-134 expression in the acute ischemic stroke patients. BMC Neurol. 2018;181:198.

20. Liu W, Li R, Yin J, Guo S, Chen Y, Fan H, et al. Mesenchymal stem cells alleviate the early brain injury of subarachnoid hemorrhage partly by suppression of Notch1-dependent neuroinflammation: involvement of Botch. J Neuroinflammation. 2019;161:8.

21. Al-Tamimi YZ, Bhargava D, Orsi NM, Teraifi A, Cummings M, Ekbote UV, et al. Compartmentalisation of the inflammatory response following aneurysmal subarachnoid haemorrhage Cytokine. 2019;123:154778.

22. Wu W, Guan Y, Zhao G, Fu XJ, Guo TZ, Liu YT, et al. Elevated IL-6 and TNF-alpha Levels in Cerebrospinal Fluid of Subarachnoid Hemorrhage Patients. Mol Neurobiol. 2016;535:3277-85.

23. Ahn SH, Savarraj JPJ, Parsha K, Hergenroeder GW, Chang TR, Kim DH, et al. Inflammation in delayed ischemia and functional outcomes after subarachnoid hemorrhage. J Neuroinflammation. 2019;161:213.

24. Li Y, Yuan L, Yang J, Lei Y, Zhang H, Xia L, et al., Changes in Serum Cytokines May Predict Therapeutic Efficacy of Tofacitinib in Rheumatoid Arthritis. Mediators Inflamm, 2019; 2019: 5617431.

25. Niwa A, Osuka K, Nakura T, Matsuo N, Watabe T, Takayasu M, Interleukin-6, MCP-1, IP-10, and MIG are sequentially expressed in cerebrospinal fluid after subarachnoid hemorrhage. $\mathrm{J}$ Neuroinflammation, 2016; 131: 217.

26. Zhang W, Sun L, Ma L, Li Z. Clinical significance of changes in IL-6, CRP and S100in serum and NO in cerebrospinal fluid insubarachnoid hemorrhage and prognosis. Exp Ther Med. 2018;162:816-20.

27. Duris K, Neuman E, Vybihal V, Juran V, Gottwaldova J, Kyr M, et al. Early Dynamics of Interleukin-6 in Cerebrospinal Fluid after Aneurysmal Subarachnoid Hemorrhage. J Neurol Surg A Cent Eur Neurosurg. 2018;792:145-51.

28. Eagles ME, Jaja BNR, Macdonald RL. Incorporating a Modified Graeb Score to the Modified Fisher Scale for Improved Risk Prediction of Delayed Cerebral Ischemia Following Aneurysmal Subarachnoid Hemorrhage. Neurosurgery. 2018;823:299-305.

29. Bache S, Rasmussen R, Rossing M, Laigaard FP, Nielsen FC, Moller K. MicroRNA Changes in Cerebrospinal Fluid After Subarachnoid Hemorrhage Stroke. 2017;489:2391-8. 
30. Vlachogiannis P, Hillered L, Khalil F, Enblad P, Ronne-Engstrom E. Interleukin-6 Levels in Cerebrospinal Fluid and Plasma in Patients with Severe Spontaneous Subarachnoid Hemorrhage. World Neurosurg. 2019;122:e612-8.

31. Schoch B, Regel JP, Wichert M, Gasser T, Volbracht L, Stolke D. Analysis of intrathecal interleukin-6 as a potential predictive factor for vasospasm in subarachnoid hemorrhage. Neurosurgery. 2007;605:828-36.

32. Hopkins SJ, McMahon CJ, Singh N, Galea J, Hoadley M, Scarth S, et al. Cerebrospinal fluid and plasma cytokines after subarachnoid haemorrhage: CSF interleukin-6 may be an early marker of infection. J Neuroinflammation. 2012;9:255.

33. Wostrack M, Reeb T, Martin J, Kehl V, Shiban E, Preuss A, et al. Shunt-dependent hydrocephalus after aneurysmal subarachnoid hemorrhage: the role of intrathecal interleukin-6. Neurocrit Care. 2014;211:78-84.

34. Chaudhry SR, Stoffel-Wagner B, Kinfe TM, Guresir E, Vatter H, Dietrich D, et al. Elevated Systemic IL-6 Levels in Patients with Aneurysmal Subarachnoid Hemorrhage Is an Unspecific Marker for Post-SAH Complications. Int J Mol Sci. 2017;1812:2580.

35. Sarrafzadeh A, Schlenk F, Gericke C, Vajkoczy P. Relevance of cerebral interleukin-6 after aneurysmal subarachnoid hemorrhage. Neurocrit Care. 2010;133:339-46.

36. Chen Y, Xia K, Chen L, Fan D. Increased Interleukin-6 Levels in the Astrocyte-Derived Exosomes of Sporadic Amyotrophic Lateral Sclerosis Patients. Front Neurosci. 2019;13:574.

\section{Figures}


Patients with $\mathrm{SAH}(\mathrm{n}=224)$

107 patients excluded

1. Admission more than $24 \mathrm{~h}$ after aSAH $(n=19)$.

2. Non-aneurysmal SAH $(n=31)$.

3. Liver, kidney, heart or lung insufficiency or infectious diseases $(n=18)$.

4. Rebleeding after admission $(n=7)$.

5.Poor prognosis without any intervention $(n=9)$.

6. Refusal of participation $(n=12)$.

7.Loss of follow up or withdrawal of study $(n=7)$.

8. Not enough sample volume $(n=4)$.

117 patients finally included in this study

$\downarrow$

Patients with good outcome $(n=78)$
Patients with poor outcome $(n=39)$

Figure 1

Flow chart of the study design. 

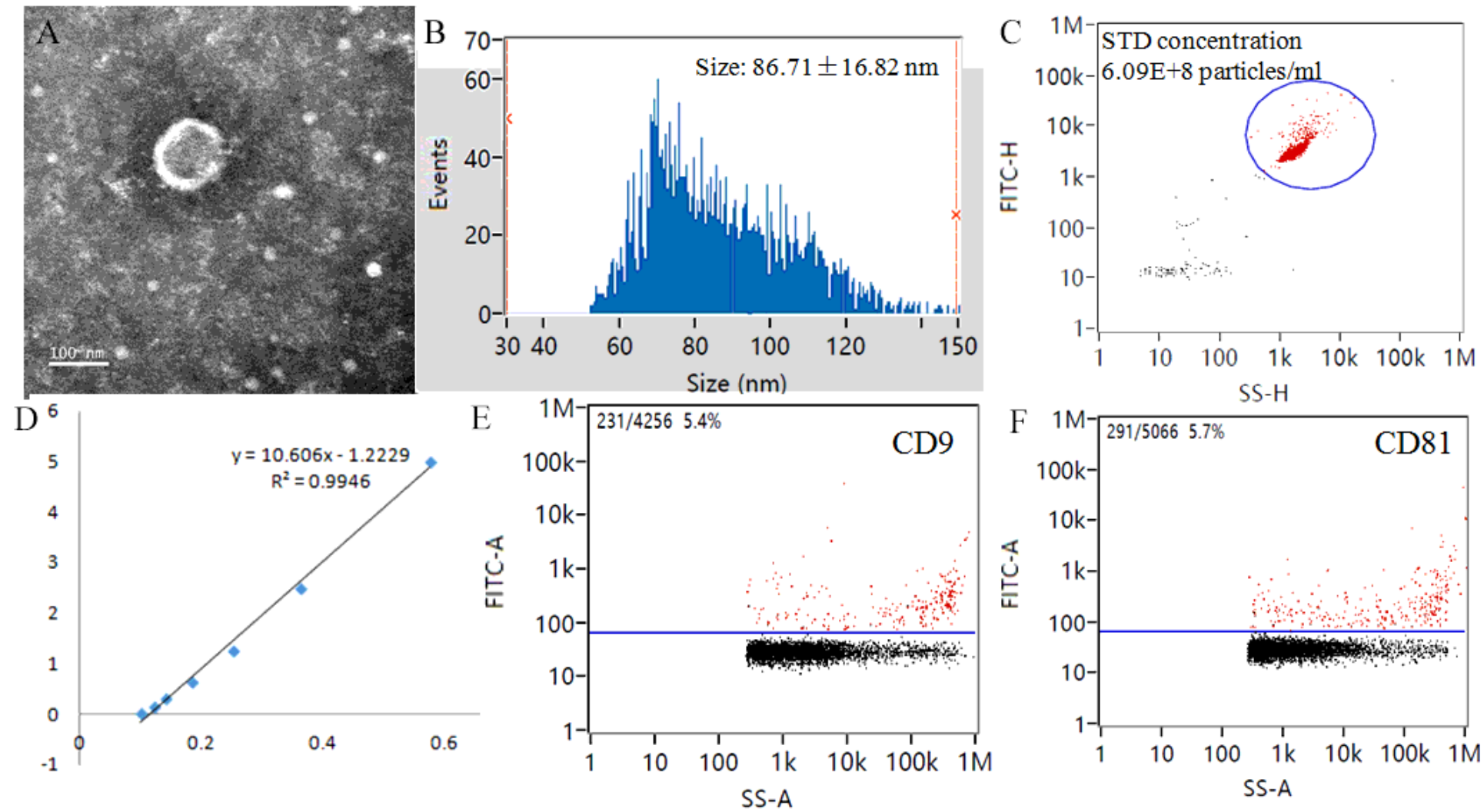

\section{Figure 2}

Characterization of Exosomes. (A, B) Transmission electron microscopy of isolated plasma exosomes. Bar, $100 \mathrm{~nm}$. (C) NanoFCM analysis of exosome concentration. (D) BCA method calculate exosome protein concentration at OD $560 \mathrm{~nm}$. (E, F) Determine the positive rate of surface protein CD9 and CD81. 


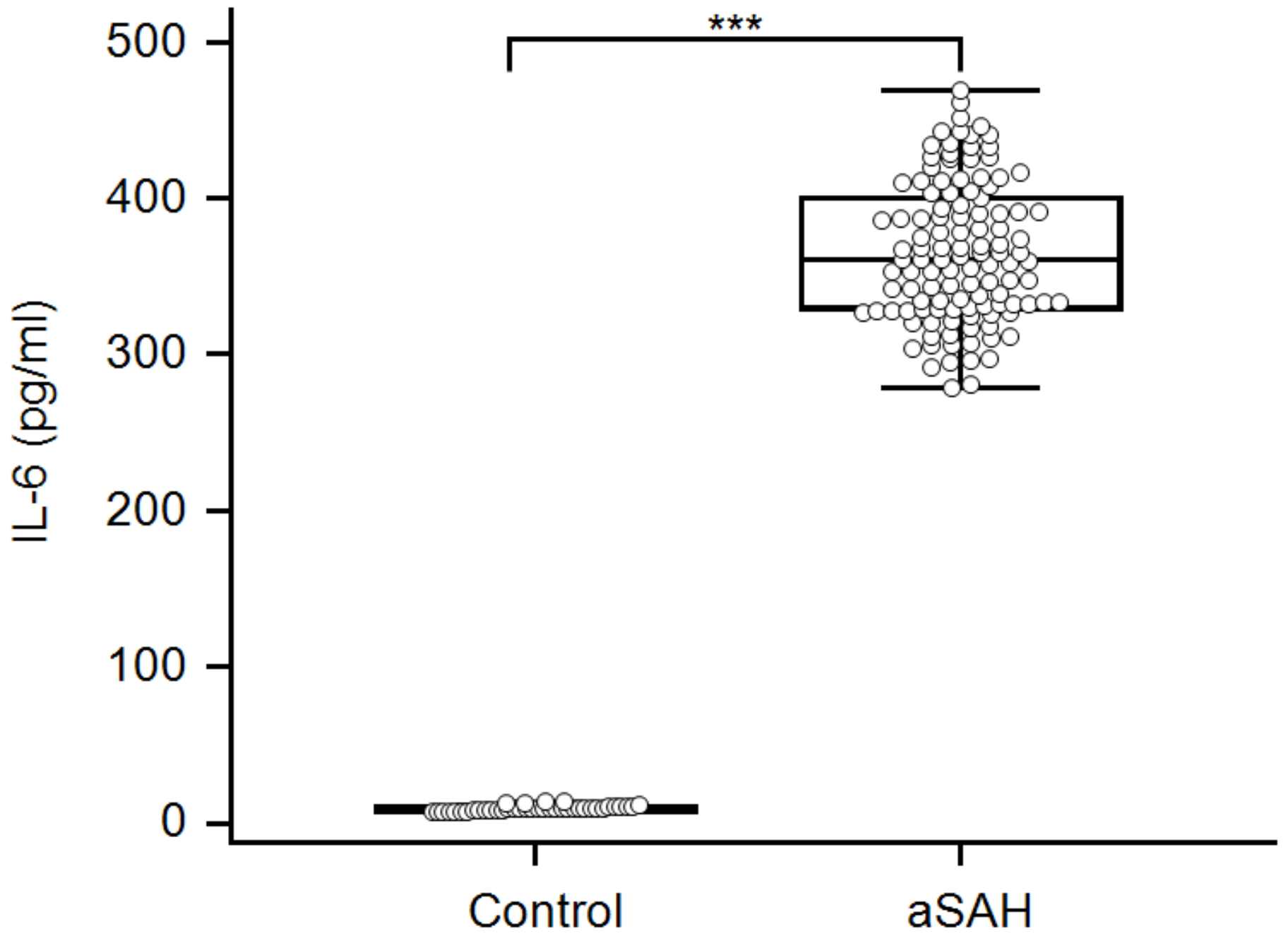

Figure 3

Characterization of Exosomes. (A, B) Transmission electron microscopy of isolated plasma exosomes. Bar, $100 \mathrm{~nm}$. (C) NanoFCM analysis of exosome concentration. (D) BCA method calculate exosome protein concentration at OD $560 \mathrm{~nm}$. (E, F) Determine the positive rate of surface protein CD9 and CD81. 

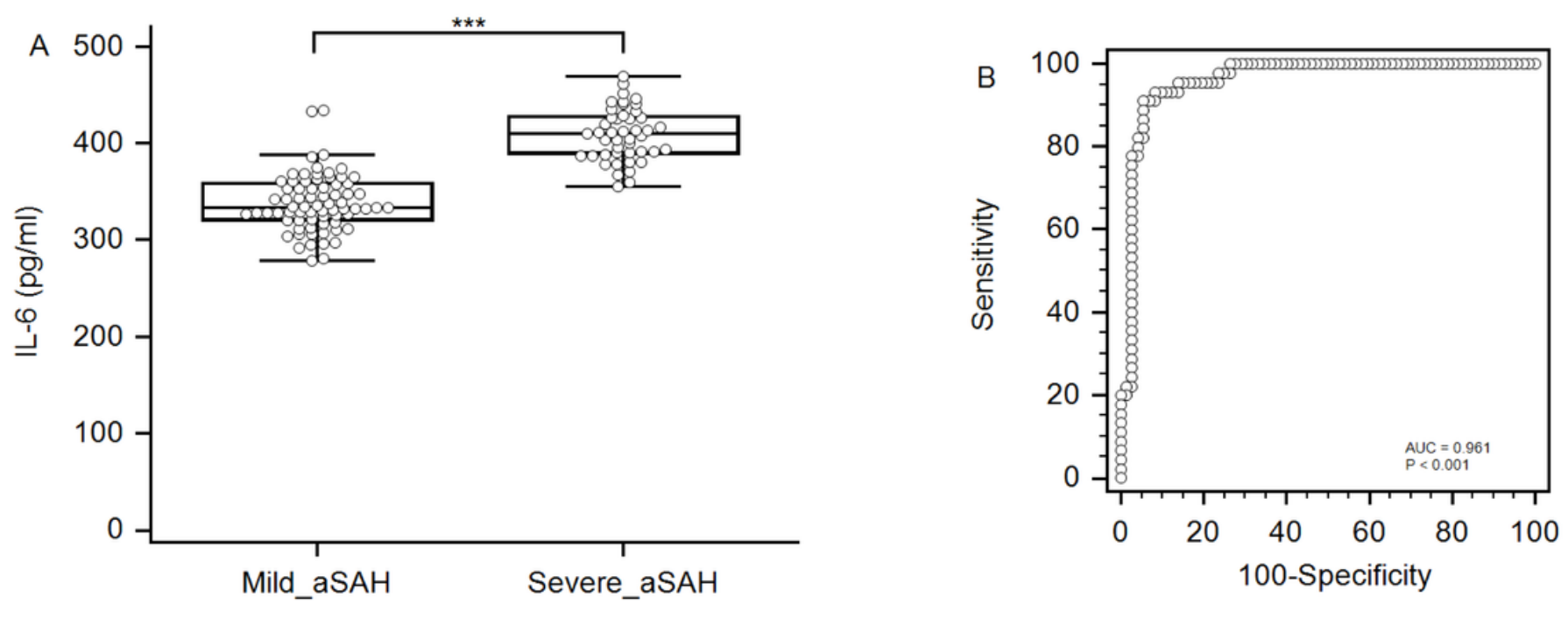

Figure 4

(A)Relative levels of IL-6NDSEVs in aSAH patients with severe aSAH and those with mild aSAH. (B)ROC curves to distinguish severe from mild aSAH patients. ${ }^{* *} \mathrm{P}<0.001$.
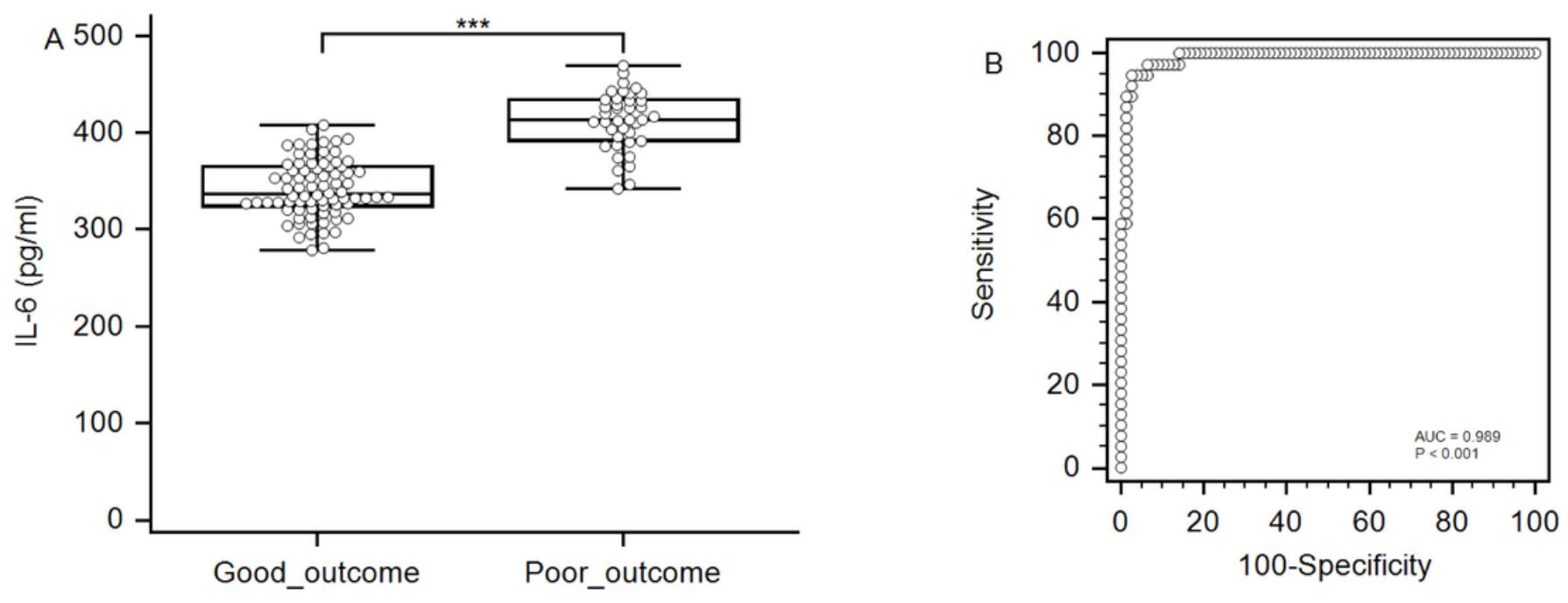

Figure 5

Relative levels of IL-6NDSEVs in aSAH patients with good outcome and those with poor outcome. $\star \star \star P<0.001$. 